\title{
Subclinical joint inflammation in patients with psoriasis without concomitant psoriatic arthritis: a cross-sectional and longitudinal analysis
}

\author{
Francesca Faustini, ${ }^{1,2}$ David Simon, ${ }^{1}$ Isabelle Oliveira, ${ }^{1}$ Arnd Kleyer, ${ }^{1}$ \\ Judith Haschka, ${ }^{1,3}$ Matthias Englbrecht, ${ }^{1}$ Alan Rodrigues Cavalcante, ${ }^{4}$ \\ Sebastian Kraus, ${ }^{1}$ Taiane Ponte Tabosa, ${ }^{4}$ Camille Figueiredo, ${ }^{1}$ Axel J Hueber, ${ }^{1}$ \\ Roland Kocijan, ${ }^{1,3}$ Alexander Cavallaro, ${ }^{5}$ Georg Schett, ${ }^{1}$ Michael Sticherling, ${ }^{6}$ \\ Jürgen Rech ${ }^{1}$
}

Handling editor Tore K Kvien

For numbered affiliations see end of article.

\section{Correspondence to Professor Georg Schett, Department of Internal Medicine 3, Rheumatology and Immunology, University of Erlangen-Nuremberg, Ulmenweg 18, Erlangen 91054, Germany; georg.schett@uk-erlangen.de}

FF, DS and IO contributed equally to authorship of this paper.

MS and JR contributed equally to senior authorship.

Received 25 October 2015 Revised 28 December 2015 Accepted 31 January 2016 Published Online First 25 February 2016

\section{ABSTRACT}

Objectives To search for subclinical inflammatory joint disease in patients with psoriasis without psoriatic arthritis (PsA), and to determine whether such changes are associated with the later development of PsA.

Methods Eighty-five subjects without arthritis ( 55 with psoriasis and 30 healthy controls) received high field MRI of the hand. MRI scans were scored for synovitis, osteitis, tenosynovitis and periarticular inflammation according to the PsAMRIS method. Patients with psoriasis additionally received complete clinical investigation, highresolution peripheral quantitative $\mathrm{CT}$ for detecting erosions and enthesiophytes and were followed up for at least 1 year for the development of PsA.

Results $47 \%$ of patients with psoriasis showed at least one inflammatory lesion on MRI. Synovitis was the most prevalent inflammatory lesion (38\%), while osteitis $(11 \%)$, tenosynovitis (4\%) and periarticular inflammation $(4 \%)$ were less frequent. The mean $( \pm$ SD) PsAMRIS synovitis score was 3.0 2.5 units. Enthesiophytes and bone erosions were not different between patients with psoriasis with or without inflammatory MRI changes. The risk for developing PsA was as high as $60 \%$ if patients had subclinical synovitis and symptoms related to arthralgia, but only $13 \%$ if patients had normal MRIs and did not report arthralgia.

Conclusions Prevalence of subclinical inflammatory lesions is high in patients with cutaneous psoriasis. Arthralgia in conjunction with MRI synovitis constitutes a high-risk constellation for the development of PSA.

\section{INTRODUCTION}

Psoriasis is a chronic inflammatory disease affecting $2-3 \%$ of the population. ${ }^{1}$ Between $15 \%$ and $30 \%$ of patients with psoriasis develop psoriatic arthritis (PsA), which can manifest as peripheral arthritis, enthesitis or axial disease. ${ }^{2}$ Usually musculoskeletal symptoms start after the onset of skin disease. Hence, in the majority of cases (60\%) skin disease antedates arthritis. ${ }^{3}$ Development of the Classification of Psoriatic Arthritis (CASPAR) classification criteria for PsA has greatly helped to better characterise musculoskeletal involvement in patients with psoriasis, especially identifying those cases having developed clinical manifestations of inflammatory musculoskeletal disease. ${ }^{4}$
The fact that psoriatic skin disease has a higher prevalence than arthritis raises the question whether patients with psoriasis without PsA are indeed spared from joint inflammation or whether mild changes, which escape physical examination, can be found in some patients. If the latter is true, a subgroup of patients with psoriasis may be characterised by subclinical inflammation, which has been earlier recognised as 'occult PsA'. Indeed, ultrasound examination of the joints has recently uncovered inflammatory lesions in patients with psoriasis. ${ }^{6}$ Hence, detecting subclinical joint inflammation could allow better understanding of the transition from skin to joint disease and therefore help to identify patients, who would benefit from more early treatment of the disease.

We recently described that patients with psoriasis without PsA exhibit enthesiophytes as the result of pathological bone formation in the joints. ${ }^{7}$ Bone formation is a pathological hallmark of PsA. The presence of similar changes in patients with psoriasis strongly supports the hypothesis of subclinical joint pathology that antedates the clinical onset of PsA. These structural lesions also highlight the well described entheseal pathology in patients with PsA. ${ }^{8-14}$ It is still unclear, however, to which extent inflammatory changes in the joints precede the onset of PsA, how such changes are related to structural pathology and whether they influence the progression of psoriasis to PsA. ${ }^{15}$

The present study was undertaken to define the prevalence and degree of inflammatory changes in the hand joints of patients with psoriasis without PsA using MRI. To explore whether inflammatory changes are related to structural changes in the joints we performed high-resolution peripheral quantitative CT (HR-pQCT). Finally we addressed whether subclinical inflammation influences the progression from psoriasis to PsA.

\section{METHODS \\ Patients}

Patients with psoriasis $(\mathrm{N}=70)$ without previous diagnosis of PsA were consecutively referred from the Dermatology to the Rheumatology Clinic of the University of Erlangen-Nuremberg for detailed clinical examination for musculoskeletal disease. 
Fifteen patients were classified as having PsA according to CASPAR criteria at the initial investigation and were excluded from further analysis. The residual 55 patients with patients with no past or present signs of PsA received a 1.5 Tesla MRI scan as well as an HR-pQCT scan of the dominant hand. In addition, 30 healthy controls without psoriasis and with no signs of musculoskeletal disease received MRI scans of the dominant hand to evaluate the 'background noise' of MRI changes in healthy volunteers. The study was conducted upon approval of the local ethics committee of the University of Erlangen and with the authorisation of the National Radiation Safety Agency (Bundesamt für Strahlenschutz). Patients participated after signing informed consent.

\section{Clinical examination}

Demographic characteristics including age, sex, body mass index (BMI) and smoking status were recorded. Duration and severity (psoriasis area severity index (PASI) score) of psoriasis were documented by the dermatologist (MS). ${ }^{16}$ Furthermore, psoriasis subtype, scalp and nail involvement and the Dermatology Life Quality Index (DLQI) questionnaire were recorded. ${ }^{17}$ All patients received clinical investigation by an experienced rheumatologist (FF) for the presence of musculoskeletal disease including tender joint count (TJC) 68, swollen joint count 66, visual analogue scale (VAS) for pain and VAS for the patients global condition. All patients were additionally checked for the presence of enthesitis, dactylitis and inflammatory back pain. If signs of inflammation such as clinical synovitis (swelling), enthesitis or dactylitis were present patients were excluded. The health assessment questionnaire (HAQ) was assessed to document physical function. Furthermore, current anti-inflammatory treatment for psoriasis was documented. Inflammatory and immunological markers such as $\mathrm{C}$ reactive protein (CRP), rheumatoid factor and anticyclic citrullinated peptide antibodies were measured. Patients with psoriasis were followed over at least 1 year to check for the transition to PsA defined as to fulfil the CASPAR criteria.

\section{MRI}

MRI scans of the dominant hand were performed with a $1.5 \mathrm{~T}$ Magneton Avanto system (Siemens, Erlangen, Germany). Patients were examined in a prone position with the hand to be imaged stretched and overhead. T1-weighted axial images (voxel size $0.5 \times 0.5 \times 3 \mathrm{~mm}$, field-of-view (FOV) $150 \mathrm{~mm}$, echo time (TE): $13 \mathrm{~ms}$, repetition time (TR): $766 \mathrm{~ms}$, slice thickness: $3 \mathrm{~mm})$ after intravenous gadolinium injection $(0.2 \mathrm{~mL} / \mathrm{kg})$ were used to assess synovitis. The same T1-weighted images were assessed to evaluate tenosynovitis and periarticular inflammation along with coronal images for confirmation of the findings on a perpendicular plane. T2-weighted coronal fat saturated (turbo inversion recovery magnitude (TIRM)) sequences (voxel size $0.5 \times 0.5 \times 2.5 \mathrm{~mm}$, FOV $220 \mathrm{~mm}$, TE: $60 \mathrm{~ms}$, TR: $3500 \mathrm{~ms}$, slice thickness $2.5 \mathrm{~mm}$ ) were used to assess osteitis.

\section{MRI analysis}

Image analysis focused on the metacarpophalangeal (MCP), proximal interphalangeal (PIP) and distal interphalangeal (DIP) joints of the second to fifth fingers. Synovitis and osteitis were assessed according to the Outcome Measures in Rheumatology Clinical Trials (OMERACT) pathology definitions and scored by using the PsA MRI scoring system (PsAMRIS). ${ }^{17}$ According to the scoring system for synovitis and flexor tenosynovitis the entire joint was assessed, for osteitis the proximal and distal part of each joint were evaluated separately and for periarticular inflammation volar and dorsal aspects of the entire joint were evaluated. ${ }^{18}$ We assigned a score to synovitis on a $0-3$ scale, which includes absent, mild, moderate or severe thickening of the synovial membrane by thirds of the maximum potential volume of the enhancing synovium. ${ }^{18}$ Similarly the grading of osteitis on a 0-3 scale was correspondent to thirds of the assessed bone volume showing increased water content. ${ }^{18}$ Flexor tendon tenosynovitis was graded $0-3$ on the basis of the thickening of the tendon sheath measured by half of the maximum thickness of the enhancing tendon sheath synovium. Periarticular inflammation was just graded as present or absent. ${ }^{18}$ The analysis was conducted per patient, all images were evaluated by two blinded and independent readers (DS, $\mathrm{FF})$.

\section{High-resolution peripheral quantitative CT}

All patients underwent HR-pQCT scans of the MCP joints 2 and 3 of the dominant hand through an XtremeCT scanner (SCANCO Medical, Bruetisellen, Switzerland). Image acquisition and analysis have been described in detail elsewhere. ${ }^{7}$ Two independent and blinded readers (DS, SK) examined the HR-pQCT images for the presence and extent of enthesiophytes and erosions. Enthesiophytes, bony protrusions originating from the cortical shell and found at the insertion sites of capsule and ligaments, or at the pulleys of extensor tendons, were evaluated in number and size. ${ }^{7}$ The distance between the highest surface of the enthesiophyte and the original surface of the cortical bone was defined as the size $(\mathrm{mm}){ }^{8}{ }^{19}$ Erosions, defined as breaks of the cortical shell and visible in two planes, were evaluated in their numbers and volumes. ${ }^{19}$ The volume $\left(\mathrm{mm}^{3}\right)$ assessment was obtained by applying the half-ellipsoid formula as previously described. ${ }^{20}$ Erosion volume and enthesiophyte size were assessed in the largest lesion of each quadrant (target lesion). All images were analysed and measured through the open source DICOM viewer Osirix V4.1 (Rosslyn, Virginia, USA). Three-dimensional image software provided by the manufacturer was used to obtain additional images for illustrative purposes.

\section{Statistical analysis}

To analyse data we used SPSS software for statistics (IBM SPSS 21.0, IBM Corporation, Armonk, New York, USA). Continuous variables are summarised as mean $\pm \mathrm{SD}$ while categorical variables as numbers and percentages. To compare mean values between subgroups and to explore correlations, after testing for Gaussian distribution, parametrical or non-parametrical tests were applied as appropriate. Adjustments for confounding factors such as age and disease duration were applied to partial correlation analysis as appropriate. To explore associations between categorical variables, $\chi^{2}$ test or Fisher's test were used as appropriate. To test inter-reader reliability in defining MRI pathologies in a semiquantitative manner, Kappa coefficients were calculated to assess inter-reader reliability in detecting MRI pathologies. $\mathrm{p}$ Values $<0.05$ were regarded as statistically significant.

\section{RESULTS \\ Demographic and clinical features of the patients with psoriasis}

Fifty-five patients with psoriasis (35 men and 20 women) underwent MRI and HR-pQCT imaging. Demographic and clinical characteristics of these patients are shown in table 1 . Briefly, patients with psoriasis showed a mean $( \pm S D)$ age of $49.0 \pm 11.4$ years, and disease duration of $15.2 \pm 15.4$ years. The 


\begin{tabular}{|c|c|}
\hline & $N=55$ \\
\hline \multicolumn{2}{|l|}{ Demographic characteristics } \\
\hline Sex, male/female, $\mathrm{N}$ & $35 / 20$ \\
\hline Age, years $($ mean $\pm S D)$ & $49.0 \pm 11.4$ \\
\hline Height, $\mathrm{cm}($ mean \pm SD) & $174.2 \pm 9.3$ \\
\hline Weight (kg) & $84.9 \pm 20.1$ \\
\hline Body mass index, units $(m e a n \pm S D)$ & $27.9 \pm 5.6$ \\
\hline Smokers, N (\%) & $16(29.1)$ \\
\hline \multicolumn{2}{|l|}{ Characteristics of psoriatic skin disease } \\
\hline Disease duration, years $(m e a n \pm S D)$ & $15.2 \pm 15.4$ \\
\hline Psoriasis vulgaris, $\mathrm{N}(\%)$ & $40(72.7)$ \\
\hline Other subtypes, N (\%) & $15(27.3)$ \\
\hline Nail involvement, $\mathrm{N}(\%)$ & $28(50.9)$ \\
\hline Scalp involvement, $\mathrm{N}(\%)$ & $16(29.1)$ \\
\hline PASI score, units (mean $\pm S D)$ & $6.2 \pm 8.0$ \\
\hline DLQI score, units (mean \pm SD) & $6.3 \pm 6.1$ \\
\hline \multicolumn{2}{|l|}{ Musculoskeletal symptoms } \\
\hline Tender joint count $68, \mathrm{~N}\left(\mathrm{mean}_{ \pm} \mathrm{SD}\right)$ & $1.6 \pm 2.9$ \\
\hline Tender joint count $>0, \mathrm{~N}$ & 21 \\
\hline Swollen joint count $66, \mathrm{~N}$ & 0 \\
\hline Presence of enthesitis, $\mathrm{N}$ & 0 \\
\hline Presence of dactylitis, N & 0 \\
\hline VAS pain, mm $($ mean \pm SD) & $25.6 \pm 27.0$ \\
\hline VAS global, mm (mean \pm SD) & $23.0 \pm 25.5$ \\
\hline HAQ score (units, mean \pm SD) & $0.4 \pm 0.5$ \\
\hline \multicolumn{2}{|l|}{ Laboratory parameters } \\
\hline C reactive protein, $\mathrm{mg} / \mathrm{L}(\text { mean } \pm \mathrm{SD})^{*}$ & $3.8 \pm 4.6$ \\
\hline ACPA positive, $\mathrm{N}$ & 0 \\
\hline RF positive, $\mathrm{N}(\%) \dagger$ & $4(7.3)$ \\
\hline \multicolumn{2}{|l|}{ Treatment modalities } \\
\hline No current treatment, $\mathrm{N}(\%)$ & $15(27.3)$ \\
\hline Topical treatments $\mathrm{N}(\%)$ & $25(45.5)$ \\
\hline Fumaric acid $\mathrm{N}(\%)$ & $11(20.0)$ \\
\hline Methotrexate and other systemic agents $\mathrm{N}(\%)$ & $9(16.4)$ \\
\hline TNF inhibitors $\mathrm{N}(\%)$ & $3(5.5)$ \\
\hline IL-12/LL-23 inhibitors N (\%) & $1(1.8)$ \\
\hline
\end{tabular}

*Normal value $<5 \mathrm{mg} / \mathrm{mL}$.

tAll four patients had low-titre RF $(<50 \mathrm{IE} / \mathrm{mL})$.

ACPA, anticyclic citrullinated peptide antibodies; DLQI, Dermatology Life Quality Index $\mathrm{HAQ}$, health assessment questionnaire; IL, interleukin; PASI, psoriasis area severity index; RF, rheumatoid factor; TNF, tumour necrosis factor; VAS, visual analogue scale.

most common psoriasis subtype was plaque psoriasis $(72.7 \%)$. Nail and scalp involvement were present in $50.9 \%$ and $29.1 \%$, respectively. Disease severity was moderate with a mean $( \pm S D)$ PASI score of $6.2 \pm 8.0$, moderately impacting patients' quality of life (DLQI score: 6.3 \pm 6.1 ). None of the patients had signs of joint swelling, while some reported tenderness $(38 \%$; TJC $1.6 \pm 2.9)$, pain $(57 \%$; VAS pain $25.6 \pm 27.0)$ or impaired function $(53 \%$; HAQ score $0.4 \pm 0.5)$. CRP levels were within the normal range. Details on treatment regimens are indicated in table 1 .

\section{Inflammatory MRI changes in patients with psoriasis}

Prevalence of MRI inflammatory lesions in patients with cutaneous psoriasis was high. Hence, 26 out of the 55 patients $(47.3 \%)$ showed at least one sign of MRI inflammation. In detail 21 (38.2\%) patients showed synovitis, 6 (10.9\%) osteitis,
$2(3.6 \%)$ presented with flexor tendon tenosynovitis and 2 (3.6\%) periarticular inflammation (figure 1 and table 2).

Synovitis was most pronounced in the MCP and PIP joints with MCP involvement in 29.1\%, PIP involvement in $23.6 \%$ and DIP involvement in $3.6 \%$ of the patients (figure 1). Signs of osteitis were detected in $7.3 \%$ of the patients in the MCP joints, $1.8 \%$ in the PIP joints and $3.6 \%$ in the DIP joints. Tenosynovitis was detected in two patients, one of them involving the flexor tendon of the fourth digit and in one the fifth digit. In both cases, thickening and effusion affected the entire tendon sheath (figure 2). Periarticular inflammation was found in the PIPs and DIPs in two patients and resembled bright appearance of the periosteal area and the periarticular soft tissues.

According to the PsAMRIS scoring system, the extent of synovitis in patients with MRI lesions was 3.0 2.5 units, for osteitis it was $1.8 \pm 1.0$ units, for tenosynovitis $10.5 \pm 2.1$ units and for periarticular inflammation $3.0 \pm 1.4$ units. If present, synovitis was mild to moderate: None of the joints scored 3 (severe), while only five scored 2 (moderate) and the remaining were scored 1 (mild synovitis). Interobserver agreement for the detection of synovitis, osteitis, tenosynovitis and periarticular inflammation ranged from substantial to almost perfect. Kappa coefficient accounted for 0.91 for synovitis $(p<0.001), 0.68$ for osteitis $(p<0.001), 0.80$ for tenosynovitis $(p<0.001)$ and 0.82 $(\mathrm{p}<0.001)$ for periarticular inflammation.

\section{Comparison of MRI findings in patients with psoriasis and healthy controls}

In contrast to patients with psoriasis the prevalence of inflammatory MRI changes in the healthy control group was low. Only 4 out of 30 subjects (13.3\%) displayed at least one sign of inflammation. Synovitis was found in two patients $(6.7 \%)$, osteitis and periarticular inflammation were each found in three patients (10.0\%), while tenosynovitis was absent (table 2). Prevalence of inflammatory changes was significantly higher in patients with psoriasis than in healthy controls $\left(\chi^{2}(1)=9.791\right.$, $\mathrm{p}=0.002)$. This difference was attributable to synovitis $\left(\chi^{2}(1)\right.$ $=9.768, \mathrm{p}=0.002)$, while the prevalence of osteitis, tenosynovitis and periarticular inflammation were not statistically different.

\section{Relation of MRI inflammation to clinical and demographic characteristics}

We next tested whether subclinical inflammation in patients with psoriasis is related to specific clinical characteristics. Patients presenting with inflammatory lesions (either synovitis, osteitis, tenosynovitis or periarticular inflammation) were significantly older than those without lesions $(51.9 \pm 10.9$ years vs $46.6 \pm 11.4$ years; $U=258.5, Z=-2.001, p=0.045)$.). Similar age differences were found in the presence or absence of synovitis $(52.3 \pm 11.6$ years vs $47.0 \pm 11.0$ years; $p=0.049)$. Furthermore, presence of inflammation in the MRI was associated with smoking exposure $\left(\chi^{2}(1)=13.13, p=0.001\right)$, while no sex differences were found. Also the extent and duration of psoriasis, the presence or absence of nail or scalp involvement and the BMI at the time of the imaging were not different between patients with or without MRI signs of inflammation.

\section{Relation of MRI inflammation to structural bone changes}

To address potential associations between inflammation and bone microstructural changes we compared inflammatory changes in the MRI to structural changes obtained by HR-pQCT. As reported previously, ${ }^{6}$ enthesiophytes were the dominant structural lesion in patients with psoriasis (table 2). 
A

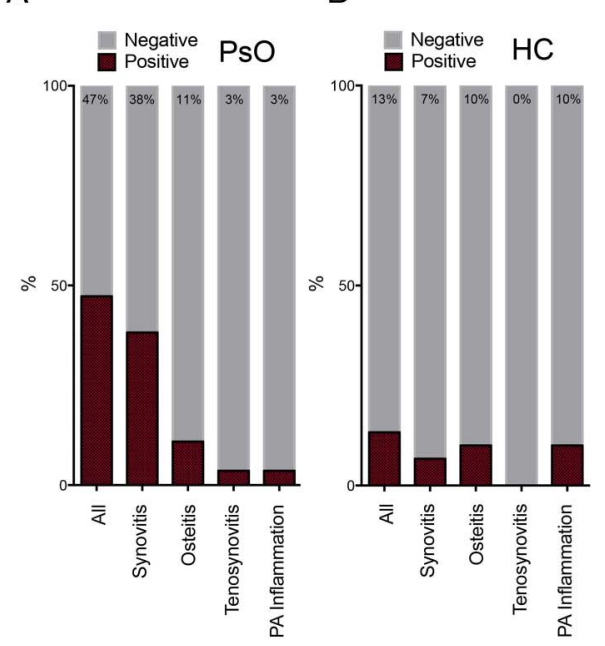

C
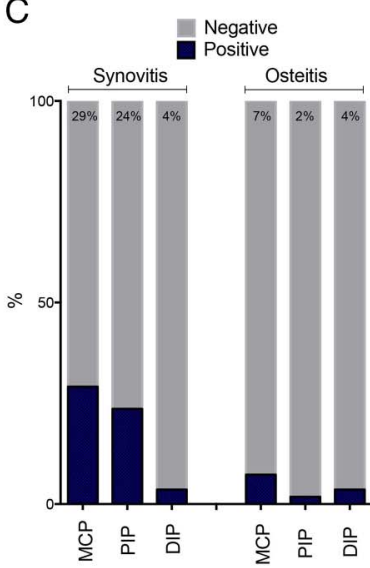

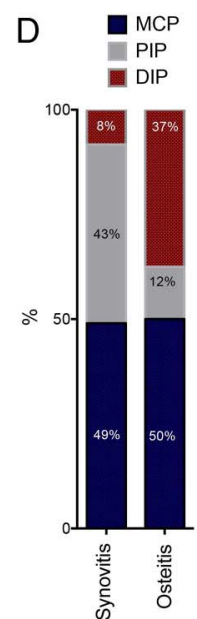

$\mathrm{E}$

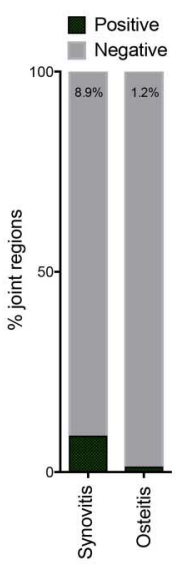

Figure 1 Frequency, distribution and extent of joint inflammation in patients with psoriasis. (A and B) Number of patients with psoriasis (A) and healthy controls (B) with signs of inflammatory changes in the metacarpophalangeal (MCP), proximal interphalangeal (PIP) and distal interphalangeal (DIP) joints in the MRI. (C) Number of patients with psoriasis with synovitis and osteitis in the equivalent joint regions. (D) Anatomical distribution of synovitis and osteitis, if present, in patients with psoriasis. (E) Percentage of joint regions afflicted by synovitis or osteitis of all joint regions.

While an average number of 0.5 erosions were found in patients with psoriasis (mean \pm SD: $0.49 \pm 0.94$ ), the average number of enthesiophytes exceeded 5 lesions per patient (5.62 23.30$)$. Erosions were typically localised in the metacarpal heads, while enthesiophytes were more evenly distributed among the metacarpal heads and the phalangeal bases. Patients with psoriasis with MRI inflammation did not show higher numbers $(5.7 \pm 3.6$ vs $5.6 \pm 3.1, \mathrm{p}=0.86)$ or increased size $(4.0 \pm 2.5$ vs $4.0 \pm 2.0$, $\mathrm{p}=0.73$ ) of enthesiophytes compared with patients without inflammation. Furthermore, no significant correlation between

Table 2 Prevalence of inflammatory MRI and structural CT lesions

(A) Prevalence of inflammatory MRI lesions in patients with psoriasis and healthy controls

\begin{tabular}{|llll|} 
& $\begin{array}{l}\text { Patients with } \\
\text { psoriasis } \\
\mathrm{N}=55\end{array}$ & $\begin{array}{l}\text { Healthy controls } \\
\mathrm{N}=30\end{array}$ & $\mathrm{p}$ Value \\
\hline Any inflammation N (\%) & $26(47)$ & $4(13)$ & 0.002 \\
\hline Synovitis N (\%) & $21(38)$ & $2(7)$ & 0.002 \\
\hline Osteitis N (\%) & $6(11)$ & $3(10)$ & 1.000 \\
\hline Periarticular inflammation N (\%) & $2(4)$ & $3(10)$ & 0.340 \\
\hline Tenosynovitis N (\%) & $2(4)$ & 0 & 0.538 \\
\hline
\end{tabular}

(B) Prevalence of structural CT lesions in patients with psoriasis Erosions Enthesiophytes

\begin{tabular}{|cll|}
\hline Lesions, N total & 27 & 306 \\
\hline N metacarpal head 2 (\%) & $15(55)$ & $113(37)$ \\
\hline N phalangeal base 2 (\%) & $1(4)$ & $64(21)$ \\
\hline N metacarpal head 3 (\%) & $10(37)$ & $58(19)$ \\
\hline N phalangeal base 3 (\%) & $1(4)$ & $113(37)$ \\
\hline Patients, N positive (\%) & $16(29)$ & $55(100)$ \\
\hline N metacarpal head 2 (\%) & $11(20)$ & $49(89)$ \\
\hline N phalangeal base 2 (\%) & $1(2)$ & $38(69)$ \\
\hline N metacarpal head 3 (\%) & $8(15)$ & $41(75)$ \\
\hline N phalangeal base 3 (\%) & $1(2)$ & $37(67)$ \\
\hline N (mean \pm SD)/patient & $0.49 \pm 0.94$ & $5.62 \pm 3.30$ \\
\hline
\end{tabular}

enthesiophytes and the extent of MRI synovitis $(r=-0.125$, $\mathrm{p}=0.38)$ and osteitis $(\mathrm{r}=-0.072, \mathrm{p}=0.62)$ was found. Similarly, no significant correlation between bone erosions and MRI synovitis $(r=-0.125, p=0.39)$ and osteitis, $(r=-0.072, p=0.62)$ was found.

MRI characteristics of patients with psoriasis developing PsA Forty-one patients with psoriasis (24 men and 17 women) completed the follow-up examination after 1 year with a mean time interval between baseline and follow-up of $426 \pm 88$ days. Twelve patients (29.6\%) developed PsA according to the CASPAR criteria. Patients with psoriasis, which developed PsA had significantly more pain, as shown by higher VAS pain scale $(17.5 \pm 20.4$ vs $44.4 \pm 30.9, \mathrm{p}=0.003)$ and joint tenderness $(1.0$ \pm 1.9 vs $4.0 \pm 4.7, \mathrm{p}=0.008)$ at baseline than those not progressing to PsA (table 3). Also, the VAS global health indicated a higher disease burden $(41.0 \pm 34.7$ vs $16.9 \pm 17.9, \mathrm{p}=0.007)$ and the HAQ a more compromised physical function $(0.8 \pm 0.8$ vs $0.2 \pm 0.4, p=0.007)$ for the patients who progressed to PsA during the follow-up. In contrast, no differences related to MRI findings were found between the groups.

We next tested whether a combination of clinical symptoms and MRI findings can predict the progression from psoriasis to PsA. Regarding MRI data 21 out of the 41 patients showed signs of synovitis or osteitis at baseline $(\mathrm{MRI}>0)$. Furthermore, with respect to clinical data, 17 patients had at least one tender joint $(\mathrm{TJC}>0)$. Patients with psoriasis with MRI synovitis and symptoms related to arthralgia had 55.5\% likelihood to develop PSA within 1 year. In contrast, only $15.3 \%$ of patients with psoriasis without signs of synovitis or arthralgia progressed to PsA (figure 3).

\section{DISCUSSION}

Our data show that a third of patients with cutaneous psoriasis exhibit subclinical inflammatory changes in the hand. We were surprised by the abundance of inflammatory lesions in our patient population, as we carefully excluded clinical signs of inflammatory joint disease. Hence, patients were recruited at the dermatology rather than rheumatology clinic avoiding bias 
Figure 2 MRI synovitis and osteitis associated with $\mathrm{CT}$ erosions in patients with psoriasis. (A and B) Axial metacarpophalangeal joints of patients with psoriasis. Dotted arrow indicates synovitis in (A), full arrow indicates tenosynovitis in (A) and osteitis in (B). (C and D) Coronal postcontrast T1-weighted MRI scans (left) showing osteitis (red arrows). Coronal high-resolution peripheral quantitative CT of the same joint region (right). Red circles indicate bone erosions. ( $\mathrm{E}$ and F) Axial postcontrast T1-weighted MRI scans (left) showing osteitis (red arrows). Axial high-resolution peripheral quantitative CT of the same joint region (right). Red circles indicate
T2-weighted (left) and postcontrast T1-weighted (right) MRI of the bone erosions.
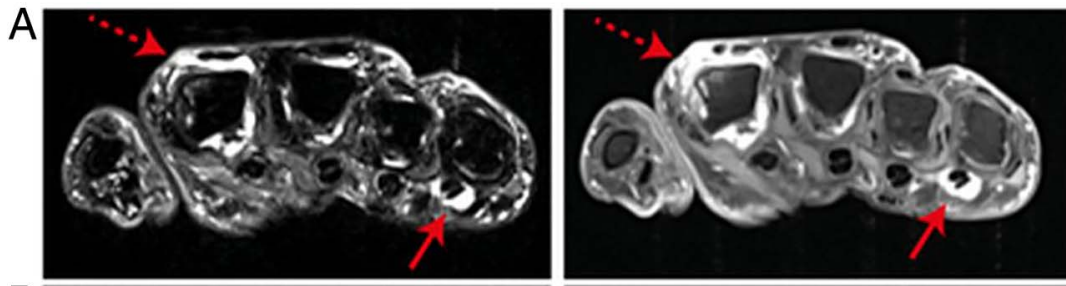

$\mathrm{B}$
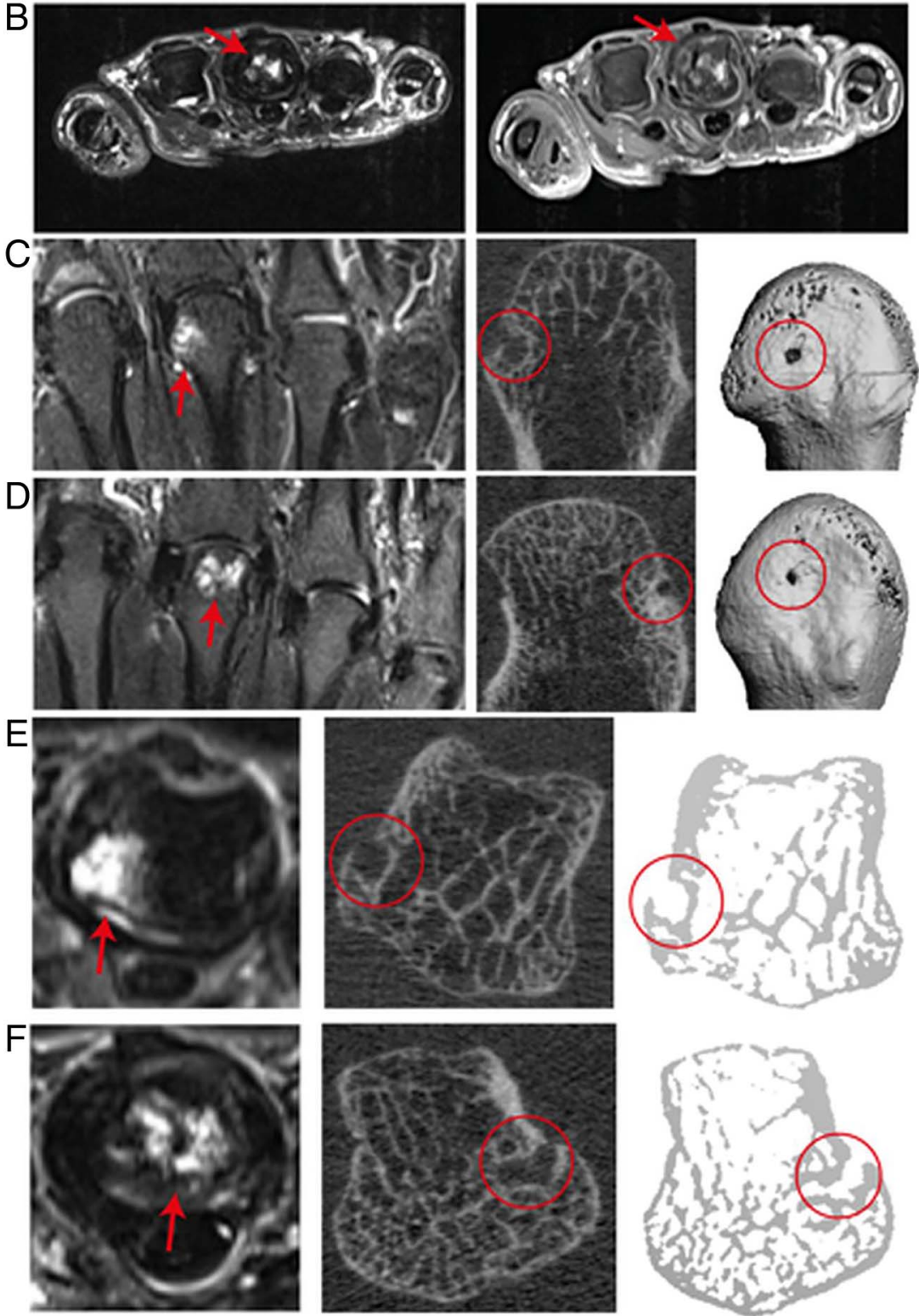

towards musculoskeletal disease. Furthermore, they received detailed musculoskeletal investigation by the rheumatologist before inclusion into the study for excluding arthritis, enthesitis and dactylitis. Nonetheless, the prevalence of subclinical inflammation was high (47\%) affecting significantly more individuals than healthy controls (13\%) as well as compared with data in healthy individuals $(27 \%)$ from recent meta-analysis. ${ }^{21}$

Few studies have yet searched for subclinical inflammation in patients with psoriasis. In an elegant ultrasound study, Naredo and colleagues showed higher prevalence of synovitis and enthesitis among patients with psoriasis compared with non-psoriatic controls. $^{6}$ The absolute frequency of inflammatory changes in this ultrasound study was lower than in our MRI study, but this might be explained by differences in the techniques and by the possibility of MRI to visualise sites not accessible by ultrasound. Two previous MRI studies in patients with psoriasis addressed the prevalence of inflammatory lesions in the knee and the foot joints. $^{22} 23$ Both studies concluded that the prevalence of inflammatory lesions is rather high in patients with psoriasis.

It is important to consider that the mere presence of inflammatory lesions in the MRI does not indicate that cutaneous psoriasis is causally linked to such lesions. In fact, neither the activity nor the duration of skin disease was associated with MRI lesions. Also, the presence of scalp involvement or nail disease, which are considered as predictors for the development of PsA ${ }^{24} 25$ were not associated with a higher burden of MRI inflammation. These findings suggest that skin and joint inflammation occur uncoupled and that skin disease may not represent 
Table 3 Clinical and MRI characteristics of patients with psoriasis with and without progression to psoriatic arthritis

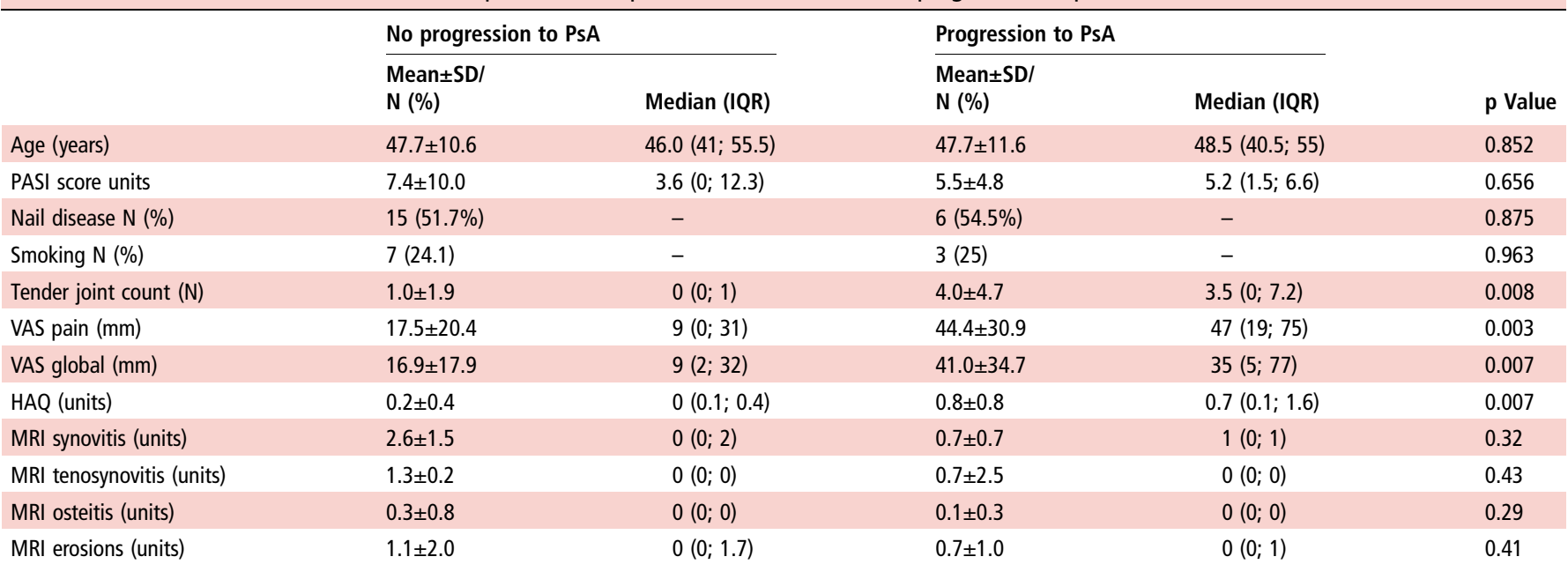

HAQ, health assessment questionnaire; PASI, psoriasis area severity index; PsA, psoriatic arthritis; VAS, visual analogue scale.

the key pacemaker for joint inflammation. In contrast, higher age and active smoking were factors determining the presence of subclinical joint inflammation in patients with psoriasis. Smoking has recently been recognised as a risk factor for incident PsA. ${ }^{26}$

Our longitudinal analysis showed that subclinical inflammation appears to substantially influence the risk of patients with psoriasis to progress to PsA. This transition to PsA is considered being a critical step in defining the onset of musculoskeletal disease in patients with psoriasis and to shape early interventions. In our longitudinal analysis we show that both symptoms related to arthralgia as well as subclinical synovitis influence the risk for progression to PsA. Hence, more than half of the patients with concomitant presence of arthralgia as well as positive MRI changes, but only $15 \%$ of patients without such changes developed PsA. Transition of psoriasis into PsA was rather high in our study as compared with other studies, ${ }^{27}$ which may be based on accumulation of more severe cases including those with beginning joint involvement in the highly specialised care setting of an University Clinic. Nonetheless, these findings indicate the possibility to define patients with psoriasis, in which preventive treatment for the development of PsA may be feasible. ${ }^{28}$ In this context it is particularly interesting that Tinazzi and colleagues also observed high progression

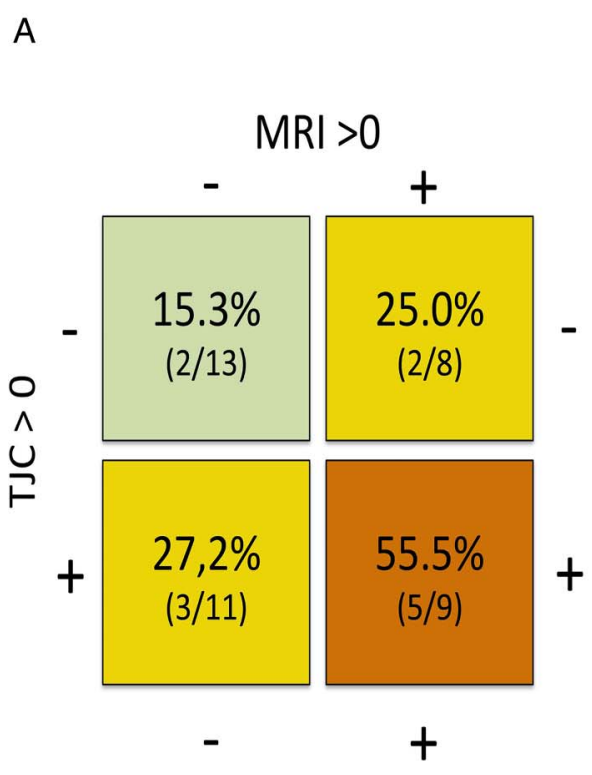

B
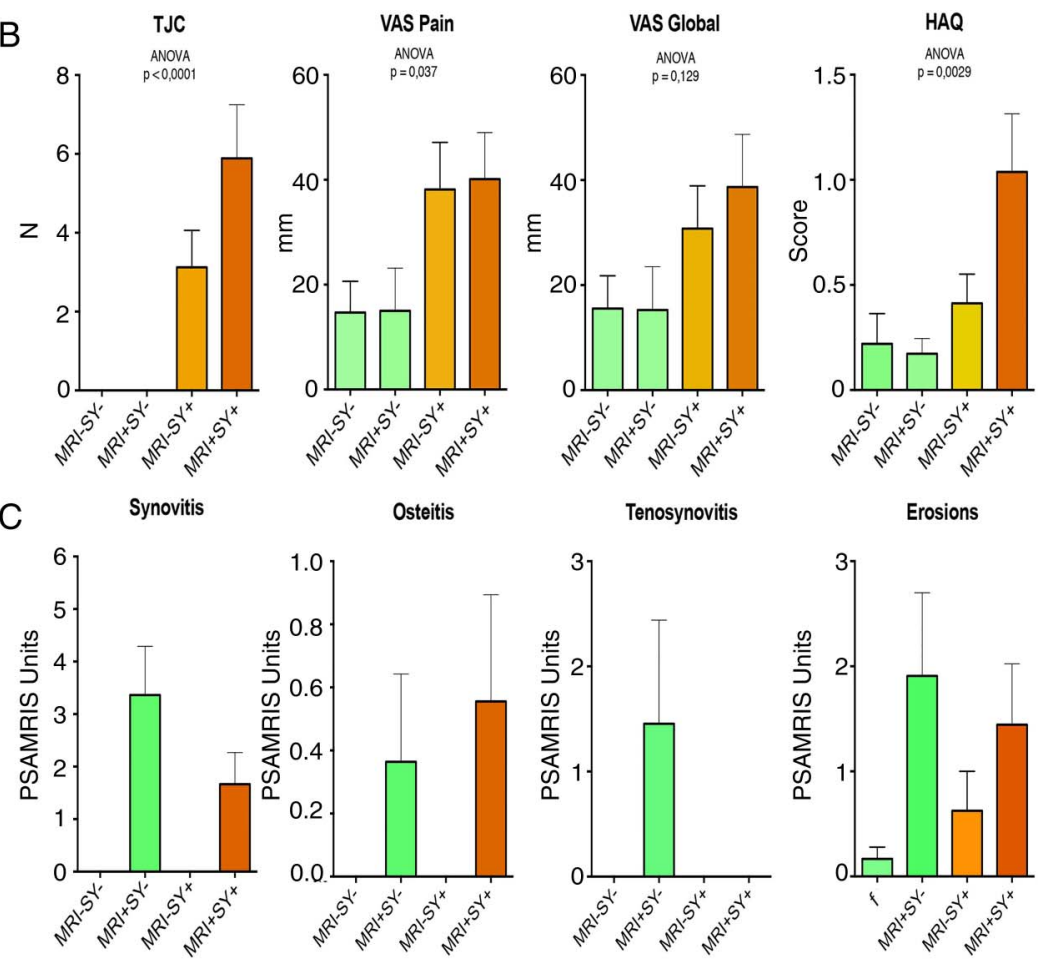

Figure 3 Risk for development of psoriatic arthritis in subgroups of patients with psoriasis. (A) Per cent risk of developing psoriatic arthritis in subgroups of patients with psoriasis based on the presence or absence of MRI findings (synovitis and/or osteitis positive; MRI $>0$ ) or joint pain (tender joint count (TJC) of 1 or more, TJC $>0$ ). (B) Clinical characteristics of the four subgroups: TJC, visual analogue (VAS) pain scale, VAS global symptoms scale and health assessment questionnaire (HAQ). (C) MRI characteristics of the four subgroups. SY, symptoms. 
rates from psoriasis to PsA in patients with entheseal abnormalities in the ultrasound. ${ }^{29}$

In summary, we provide evidence for a high prevalence of subclinical inflammation in patients with psoriasis. These findings suggest that subclinical musculoskeletal disease affects a substantial proportion of patients with psoriasis. Together with arthralgia these changes predict the risk for the onset of PsA.

\section{Author affiliations}

${ }^{1}$ Department of Internal Medicine 3, Rheumatology and Immunology, University of Erlangen-Nuremberg, Erlangen, Germany

${ }^{2}$ Clinic of Rheumatology, Karolinska University Hospital, Stockholm, Sweden

${ }^{3}$ Medical Department II, St. Vincent Hospital, The VINFORCE Study Group, Academic Teaching Hospital of Medical University of Vienna, Vienna, Austria

${ }^{4}$ CSD Clinica Som Diagnosticos, Belém, Pará, Brazil

${ }^{5}$ Department of Radiology, University of Erlangen-Nuremberg, Erlangen, Germany

${ }^{6}$ Department of Dermatology, University of Erlangen-Nuremberg, Erlangen, Germany

Contributors DS, FF, IO, AK, JH, SK, AJH, RK, MS, ARC, AC collected the data. $D S, F F, M E, I O, A J H, G S$ and JR analysed and interpreted the data. DS, FF, GS and $J R$ prepared and revised the manuscript.

Funding This study was supported by the Deutsche Forschungsgemeinschaft (SPP1468, CRC1181), the Marie Curie project OSTEOIMMUNE, the Metarthros project of the German Ministry of Science and Education, the IMI-funded project BTCure and the Pfizer Competitive Grant Award Germany.

Competing interests None declared.

Patient consent Obtained.

Ethics approval University of Erlangen-Nuremberg.

Provenance and peer review Not commissioned; externally peer reviewed.

\section{REFERENCES}

1 Mease PJ, Gladman DD, Papp KA, et al. Prevalence of rheumatologist-diagnosed psoriatic arthritis in patients with psoriasis in European/North American dermatology clinics. J Am Acad Dermatol 2013:69:729-35.

2 Villani AP, Rouzaud M, Sevrain M, et al. Prevalence of undiagnosed psoriatic arthritis among psoriasis patients: Systematic review and meta-analysis. J Am Acad Dermatol 2015;73:242-8.

3 McGonagle D, Ash Z, Dickie L, et al. The early phase of psoriatic arthritis. Ann Rheum Dis 2011;70(Suppl 1):i71-6.

4 Taylor W, Gladman D, Helliwell P, et al. Classification criteria for psoriatic arthritis: development of new criteria from a large international study. Arthritis Rheum 2006;54:2665-73.

5 Palazzi C, Lubrano E, D'Angelo S, et al. Beyond early diagnosis: occult psoriatic arthritis. J Rheumatol 2010;37:1556-8.

6 Naredo E, Möller I, de Miguel E, et al. High prevalence of ultrasonographic synovitis and enthesopathy in patients with psoriasis without psoriatic arthritis: a prospective case-control study. Rheumatology (Oxford) 2011;50:1838-48.

7 Simon D, Faustini F, Kleyer A, et al. Analysis of periarticular bone changes in patients with cutaneous psoriasis without associated psoriatic arthritis. Ann Rheum Dis Published Online First: 4 Feb 2015. doi:10.1136/annrheumdis-2014-206347

8 Finzel $\mathrm{S}$, Englbrecht $\mathrm{M}$, Engelke $\mathrm{K}$, et al. A comparative study of periarticular bone lesions in rheumatoid arthritis and psoriatic arthritis. Ann Rheum Dis 2011;70:122-7.
9 McGonagle D, Lories RJ, Tan AL, et al. The concept of a "synovio-entheseal complex" and its implications for understanding joint inflammation and damage in psoriatic arthritis and beyond. Arthritis Rheum 2007;56:2482-91.

10 Benjamin M, McGonagle D. Histopathologic changes at "synovio-entheseal complexes" suggesting a novel mechanism for synovitis in osteoarthritis and spondylarthritis. Arthritis Rheum 2007:56:3601-9.

11 Marzo-Ortega H, Tanner SF, Rhodes LA, et al. Magnetic resonance imaging in the assessment of metacarpophalangeal joint disease in early psoriatic and rheumatoid arthritis. Scand J Rheumatol 2009;38:79-83.

12 Schoellnast $\mathrm{H}$, Deutschmann HA, Hermann J, et al. Psoriatic arthritis and rheumatoid arthritis: findings in contrast-enhanced MRI. AJR Am J Roentgenol 2006;187:351-7.

13 McQueen F, Lassere M, Duer-Jensen A, et al. Testing an OMERACT MRI scoring system for peripheral psoriatic arthritis in cross-sectional and longitudinal settings. J Rheumatol 2009;36:1811-15.

14 Freeston JE, Coates LC, Nam JL, et al. Is there subclinical synovitis in early psoriatic arthritis? A clinical comparison with gray-scale and power Doppler ultrasound. Arthritis Care Res (Hoboken) 2014;66:432-9.

15 Baeten $D$, Breban $M$, Lories $R$, et al. Are spondylarthritides related but distinct conditions or a single disease with a heterogeneous phenotype? Arthritis Rheum 2013:65:12-20.

16 Fredriksson T, Pettersson U. Severe psoriasis-oral therapy with a new retinoid. Dermatologica 1978;157:238-44.

17 Finlay AY, Khan GK. Dermatology Life Quality Index (DLQI)-a simple practical measure for routine clinical use. Clin Exp Dermatol 1994;19:210-16.

18 Ostergaard M, McQueen F, Wiell C, et al. The OMERACT psoriatic arthritis magnetic resonance imaging scoring system (PsAMRIS): definitions of key pathologies, suggested MRI sequences, and preliminary scoring system for PsA Hands. J Rheumatol 2009;36:1816-24.

19 Stach CM, Bäuerle M, Englbrecht $\mathrm{M}$, et al. Periarticular bone structure in rheumatoid arthritis patients and healthy individuals assessed by high-resolution computed tomography. Arthritis Rheum 2010;62:330-9.

20 Albrecht A, Finzel S, Englbrecht $M$, et al. The structural basis of MRI bone erosions: an assessment by microCT. Ann Rheum Dis 2013;72:1351-7.

21 Mangnus L, Schoones JW, van der Helm-van Mil AHM. What is the prevalence of MRI-detected inflammation and erosions in small joints in the general population? A collation and analysis of published data. RMD Open 2015;1:e000005.

22 Emad Y, Ragab Y, Gheita T, et al. Knee enthesitis and synovitis on magnetic resonance imaging in patients with psoriasis without arthritic symptoms. J Rheumatol 2012;39:1979-86.

23 Erdem CZ, Tekin NS, Sarikaya S, et al. MR imaging features of foot involvement in patients with psoriasis. Eur J Radiol 2008;67:521-5.

24 Wilson FC, Icen M, Crowson CS, et al. Incidence and clinical predictors of psoriatic arthritis in patients with psoriasis: a population-based study. Arthritis Rheum 2009;61:233-9.

25 Langenbruch A, Radtke MA, Krensel M, et al. Nail involvement as a predictor of concomitant psoriatic arthritis in patients with psoriasis. Br J Dermatol 2014;171:1123-8.

26 Li W, Han J, Qureshi AA. Smoking and risk of incident psoriatic arthritis in US women. Ann Rheum Dis 2012;71:804-8.

27 Eder L, Haddad A, Rosen CF, et al. The incidence and risk factors for psoriatic arthritis in patients with psoriasis—a prospective cohort study. Arthritis Rheumatol Published Online First: 10 Nov 2015. doi:10.1002/art.39494

28 Ritchlin CT, Kavanaugh A, Gladman DD, et al. Treatment recommendations for psoriatic arthritis. Ann Rheum Dis 2009:68:1387-94.

29 Tinazzi I, McGonagle D, Biasi D, et al. Preliminary evidence that subclinical enthesopathy may predict psoriatic arthritis in patients with psoriasis. I Rheumatol 2011;38:2691-2. 